\title{
Exciplex Method for Remote Probing of Fuel Droplet Temperature
}

\author{
Toshikazu KADOTA**, Yoshio TANIGUCHI*** \\ and Ken KADOWAKI****
}

\begin{abstract}
Exciplex-based fluorescence thermometry was developed for remote probing of fuel droplet temperature. The liquid fuels tested were $n$-Heptane, $n$-Decane and $n$ Hexadecane which contained $1 \%$ Naphthalene and $2.5 \%$ Tetramethyl- $p$-phenylene diamine (TMPD). Also examined was unleaded regular gasoline into which no Naphthalene or TMPD was dissolved. The fuel droplet suspended at the tip of a quartz fiber was allowed to evaporate in a stream of air or gaseous Nitrogen, and its temperature was monitored using a fine thermocouple. The droplet was irradiated with a Nitrogen laser and fluorescence emission spectra were taken by means of a light collection system which consisted of a convex lens, a light stop, a double monochromator and a photomultiplier. The results showed that remote probing of the droplet temperature could be achieved by measuring the ratio of fluorescence emission intensities at two different wavelengths.
\end{abstract}

Key Words: Laser, Fluorescence Thermometry, Liquid Fuel, Evaporation, Exciplex, Temperature, Droplet

\section{Introduction}

The evaporation of fuel droplets is of great relevance to a wide variety of engineering applications including spray combustion. This resulted in a number of experimental and theoretical works carried out on this subject. Fairly complete reviews of the previous works are provided in Refs. ( 1$) \sim(4)$. It is indicated that great efforts have been directed toward the determination of droplet temperature which is one of the key factors in droplet evaporation. The temperature of an evaporating droplet rules the fuel vapor pressure, namely the fuel vapor concentration at the droplet surface. The fuel vapor concentration profile around the droplet in turn governs the rate constant of evaporation of itself as well as of neighboring droplets $^{(5)}$. The temperature measurements in the

* Received 9th August, 1990. Paper No.89-0052A

** Department of Mechanical Engineering, University of Osaka Prefecture, Mozu-Umemachi, Sakai 591, Japan

*** Mazda Motor Corporation, Fuchu-cho, Hiroshima 730-91, Japan

**** Matsue College of Technology, Matsue 690, Japan previous works have mainly been done for a stationary droplet by applying a thermocouple. This was primarily due to the difficulty in developing diagnostics for the thermometry of moving droplets.

The primary objective of the present work is the instrumentation development which is available for the remote, nonintrusive, instantaneous, point thermometry of an evaporating droplet. Among a large variety of diagnostic tools potentially applicable for this purpose, the exciplex-based fluorescence method $^{(6),(7)}$ was selected. The cross section for fluorescence in this method is generally many orders of magnitude larger than those for Raman scattering. This allows the highly time-and space-resolved measurement of droplet temperature. Since the light emitted from a droplet is shifted in frequency and hence hardly suffers from the spurious scattering of incident laser light and Mie interference. A major problem associated with this method is the quenching correction. Not much is known about this and experimental data are limited. The present work is the first to apply the exciplex-based fluorescence method for the thermometry of an evaporating droplet. This consists of the first phase of an experimental program aimed at 
providing detailed information on liquid droplets evaporating and burning within a fuel spray, and reports the measured results of the temperature of a droplet of several kinds of fuels evaporating in a uniform stream of air or Nitrogen. A fuel droplet doped with appropriate organic additives was subjected to the irradiation of a pulsed Nitrogen laser as an excitation source, and the fluorescence emission spectra from the droplet were detected.

\section{Experimental Procedure}

The principle of the method applied in the present study consists of monitoring the fluorescence emission spectra from fuel droplets doped with small amounts of appropriate organic additives which are potentially sensitive to temperature. Figure 1 gives the schematic diagram of photophysical processes which may occur in the exciplex system of Naphthalene $\left(\mathrm{N}_{\mathrm{p}}\right)$ and $N, N$, $N^{\prime}, N^{\prime}$-Tetramethyl- $p$-phenylene diamine (TMPD). The diagram is by no means complete. It contains only the processes necessary to understand the diagnostic strategies proposed. Naphthalene in the ground state $\mathrm{N}_{\mathrm{p}}^{*}$ can absorb laser light to form electronically excited Naphthalene $\mathrm{N}_{\mathrm{p}}^{*}$ It has an average lifetime of 10-100 ns, during which it may simply emit light and return to the ground state (fluorescence at the wavelength of $340 \mathrm{~nm}$ ) or collide with TMPD in the solution. $\mathrm{N}_{\mathrm{p}}^{*}$ may then return to the ground state without the emission of light (fluorescence quenching). It may transfer some of its energy to excite TMPD, and TMPD* $^{*}$ in the excited state subsequently fluoresce at the wavelength of $380 \mathrm{~nm}$. Furthermore, $\mathrm{N}_{\mathrm{p}}^{*}$ may bind reversibly with TMPD to form $\left(\mathrm{N}_{\mathrm{p}} / \mathrm{TMPD}\right)^{*}$, an exciplex (shortened from excited state complex), and $\left(\mathrm{N}_{\mathrm{p}} / \mathrm{TMPD}\right)$ * may subsequently fluoresce at the wavelength of $470 \mathrm{~nm}$. TMPD* and $\left(\mathrm{N}_{\mathrm{p}} / \mathrm{TMPD}\right)^{*}$ are at lower energies than the originally excited $\mathrm{N}_{\mathrm{p}}^{*}$ and thus their emissions are shifted toward longer wavelength than the $\mathrm{N}_{\mathrm{p}}^{*}$ emission. The above energy transfer processes, and then the fluorescence emission spec-

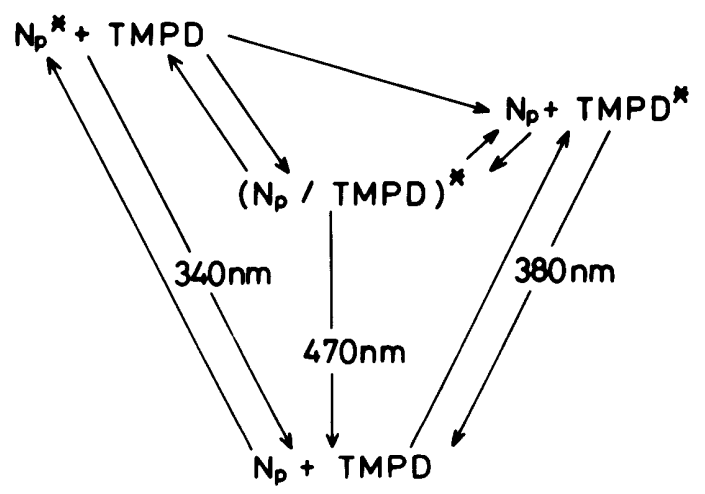

Fig. 1 Exciplex system tra are generally sensitive to temperature.

The liquid fuels tested were $n$-Heptane, $n$ Decane and $n$-Hexadecane of the special grade which were doped with $1 \%$ Naphthalene and $2.5 \%$ TMPD by mass. TMPD (Aldrich) had a stated purity of 98 $\%$ and Naphthalene was of Aldrich scintillation grade $(99+\%)$. Unleaded regular gasoline (referred to gasoline) was not doped with these additives. It may contain a priori some organic compounds which can cause temperature-sensitive fluorescence emission spectra. The preparation of the test fuel was done in Nitrogen environments to prevent TMPD from being oxidized.

Figure 2 illustrates schematically the experimental apparatus. Gaseous Nitrogen fed from a tank or air supplied by a blower was allowed to pass through a control valve, a flow meter, a heating section and a stainless steel screening mesh, and to effuse from a nozzle. The nozzle has a quadrant shape in the longitudinal cross section and the inner diameter of the exit of the nozzle is $15 \mathrm{~mm}$. With this system, a vertically upward steady free stream of air or Nitrogen with uniform velocity and temperature profiles was established in the test section above the exit of nozzle. A droplet of diameter approximately $1.8 \mathrm{~mm}$ was suspended at the tip of a quartz fiber of diameter 0.2 $\mathrm{mm}$ installed in the uniform stream of gas. A ther-

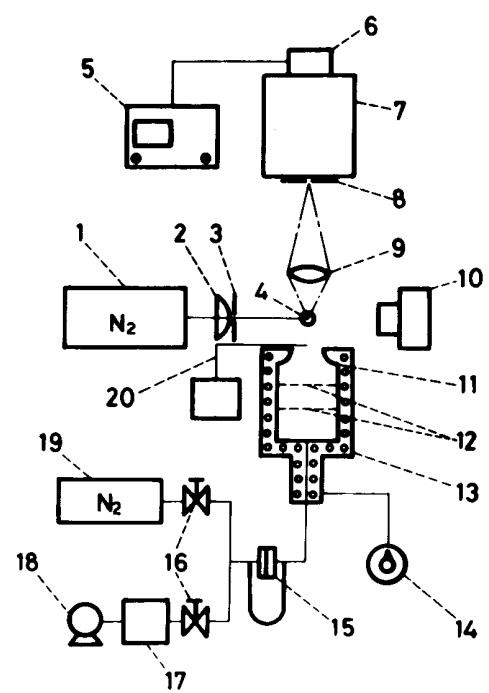

1. $\mathrm{N}_{2}$ laser 2. cylindrical lens 3. slit

4. fuel droplet 5. oscilloscope 6. PMT

7. double monochromator 8 . light stop

9. convex lens $10.35 \mathrm{~mm}$ camera 11 . nozzle

12. screening mesh 13. heater 14. volt regulator

15. flow meter 16. control valve 17. surge tank 18. blower 19. $\mathrm{N}_{2}$ tank 20. thermocouple

Fig. 2 Experimental apparatus 
mocouple of diameter $0.05 \mathrm{~mm}$ was available to measure the temperature of the droplet as well as of the gas stream.

The excitation source for all fluorescence spectra was a $1 \mathrm{MW}$ Nitrogen laser emitting monochromatic radiation at a wavelength of $337.1 \mathrm{~nm}$ with a pulse energy of $5 \mathrm{~m} \mathrm{~J} / \mathrm{p}$, a pulse width of $5 \mathrm{~ns}$ and a frequency of $1-10 \mathrm{~Hz}$. A cylindrical lens was provided to focus the incident laser light on the optical center where a fuel droplet was allowed to evaporate. An optical detection system consisted of a convex lens ( $f$ $=50.8 \mathrm{~mm}, d=60 \mathrm{~mm}$ ), a light stop of diameter 0.5 $\mathrm{mm}$, a double monochromator and a photomultiplier (Hamamatsu R928). The electrical output signal from the photomultiplier was recorded on a storage-type oscilloscope. A $35 \mathrm{~mm}$ camera was also provided for photographic observation of a fluorescing droplet.

The detailed geometry of the optical system near the droplet is illustrated in Fig. 3. The axis of the optical detection system is placed at a right angle to the incident laser light in the same horizontal plane. The fluorescence emission originating from a minute area within a circle of diameter $0.25 \mathrm{~mm}$ could only be detected by the photomultiplier. Some amounts of Naphthalene or TMPD may remain on the surface of the quartz fiber at the end of droplet evaporation and cause the alteration of fluorescence emission spectra in the following evaporation. So, the quartz fiber was burned in a flame after each test run. The preliminary test results showed that the quartz fiber did not give any appreciable effect on the measured results of fluorescence emission spectra.

Thus, a suspended fuel droplet doped with Naph-
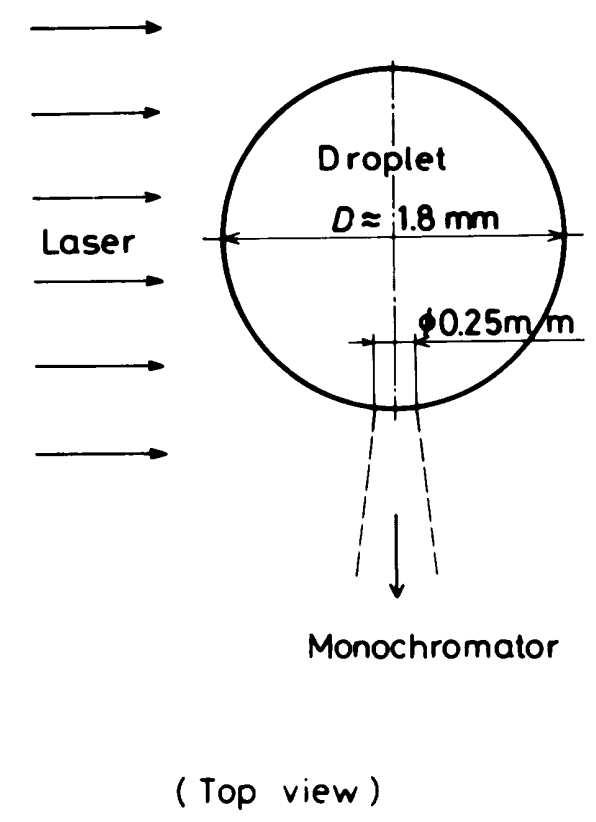

Fig. 3 Detailed geometry of optical system thalene and TMPD was allowed to evaporate in a free stream of hot air or Nitrogen at various temperatures and was subjected to Nitrogen laser excitation. The fluorescence emission spectra from a droplet of which temperature was also monitored with a fine thermocouple were measured using the optical detection system.

\section{Results}

Photographic observations were done to obtain the qualitative results of the fluorescence emission spectra from an evaporating droplet. The results showed that a hydrocarbon droplet doped with Naphthalene and TMPD fluoresced with a green color at room temperature. As the temperature was raised, the fluorescence became purple. A gasoline droplet fluoresced even with no dopant.

Figure 4 shows an example of the electrical output signal from the photomultiplier which detected the fluorescence emission at a certain wavelength from an $n$-Hexadecane droplet evaporating in a Nitrogen stream. A pulse signal has a full-width at half-maximum of several hundreds ns.

Figure 5 shows the fluorescence emission spectra from an $n$-Hexadecane droplet at two different temperatures evaporating in a Nitrogen stream. Each dot corresponds to the height of the pulse signal caused by the fluorescence emission from different droplets. All the data were obtained for the same size of droplet during steady-state evaporation. The intensity of fluorescence emission I in the ordinate of Fig. 5 is normalized with respect to the maxima of the spectra. It is evident that the spectrum for a droplet temperature of $305 \mathrm{~K}$ peaks at a wavelength of approximately $490 \mathrm{~nm}$. As the temperature is raised, the maxima are shifted toward shorter wavelength. An increase in droplet temperature also causes an increase in the

\section{$N_{2}$ Laser}

\section{INp/2.5TMPD/96.5nHexadecane in $\mathrm{N}_{2}$}

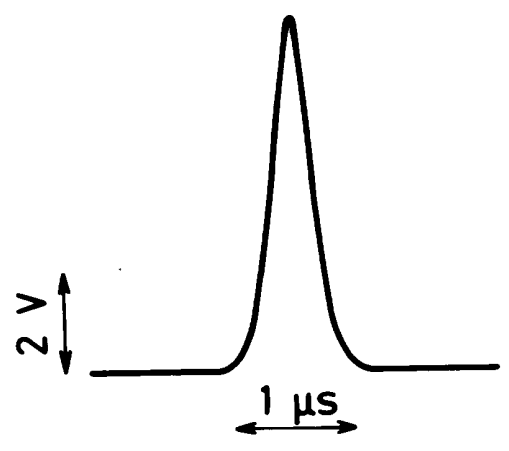

Fig. 4 Electrical output signal 
intensity at around $380 \mathrm{~nm}$. The effect of droplet temperature on the fluorescence emission spectra in Fig. 5 agrees qualitatively with the results obtained from the photographic observation, as mentioned previously.

Figure 6 shows the fluorescence emission spectra for $n$-Decane droplet evaporating in air or a Nitrogen stream. Again, the wavelength at a maximum emission intensity decreases with an increase in droplet temperature. There is a considerable difference between the spectra from a droplet in air and in Nitrogen streams. This might be partly due to the quenching effect of Oxygen dissolved in the liquid near the droplet surface. This is not conclusive yet.

The above results imply the possibility of droplet thermometry based on the measured results of fluorescence emission spectra which were found to depend on temperature. The determination of temperature can be made from the spectral band contour of the fluorescence emission spectra. This is generally done by least-squares fitting of a library of spectra determined and calibrated preliminarily at various droplet temperatures. An alternative approach, however, was

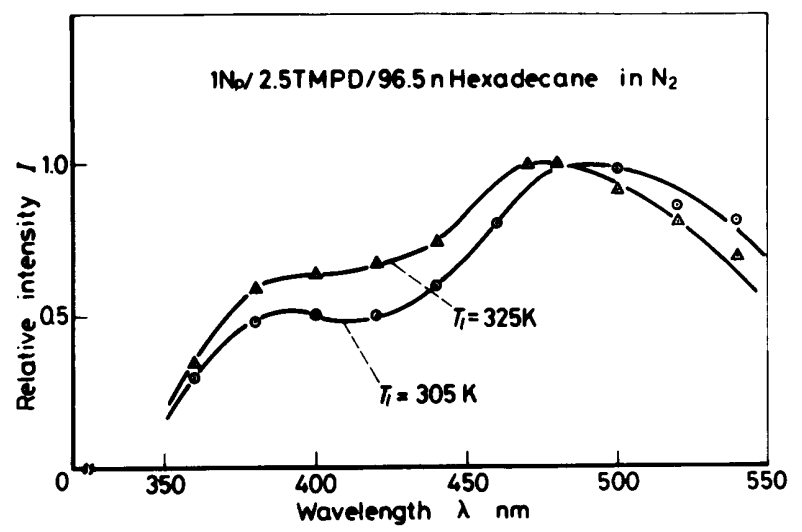

Fig. 5 Fluorescence emission spectra for $n$-Hexadecane droplet

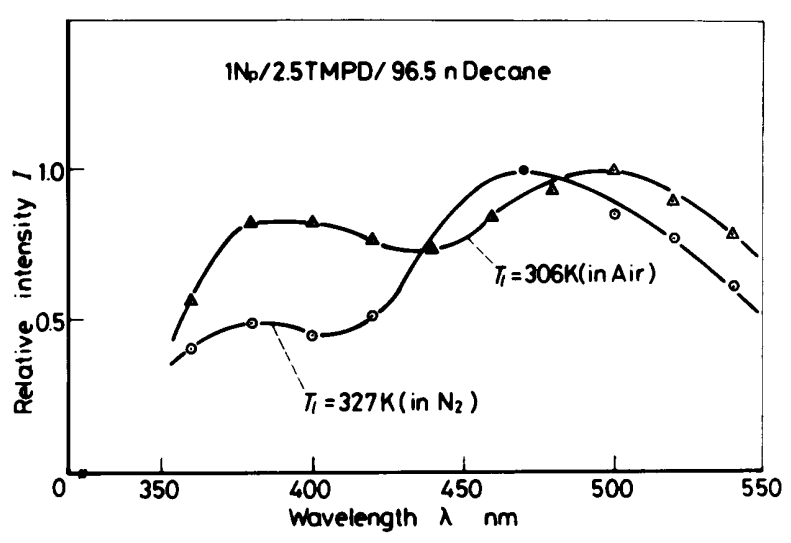

Fig. 6 Fluorescence emission spectra for $n$-Decane droplet attempted in the present study which employed the spectral-band ratio. The fluorescence emission intensities were monitored at two different wavelengths and the temperature was determined from the resultant intensity ratio.

Figure 7 shows the measured results of the intensity ratio as a function of temperature of the $n$ Hexadecane droplet evaporating in Nitrogen and air streams. $I_{1}$ and $I_{2}$ are the intensities of fluorescence emission spectra at the wavelengths of $380 \mathrm{~nm}$ and 470 $\mathrm{nm}$, respectively. It is evident that the intensity ratio increases and reaches a peak after which it decreases with an increase in the temperature of the droplet evaporating in a Nitrogen stream. The peak of the intensity ratio as a function of droplet temperature does not exist for a droplet evaporating in the air stream. It may appear when the experiments are done in an extended range of droplet temperature. The intensity ratio in Nitrogen is greater than in the air stream. It is indicated that the droplet temperature can be determined from the measured results of the intensity ratio of fluorescence emission at two different wavelengths.

The intensity ratio is a weak function of droplet temperature near the peak where the temperature measurements may include a considerable amount of error. However, it is likely that a peak of the intensity ratio disappears or the droplet temperature at the peak intensity ratio would be shifted by selecting

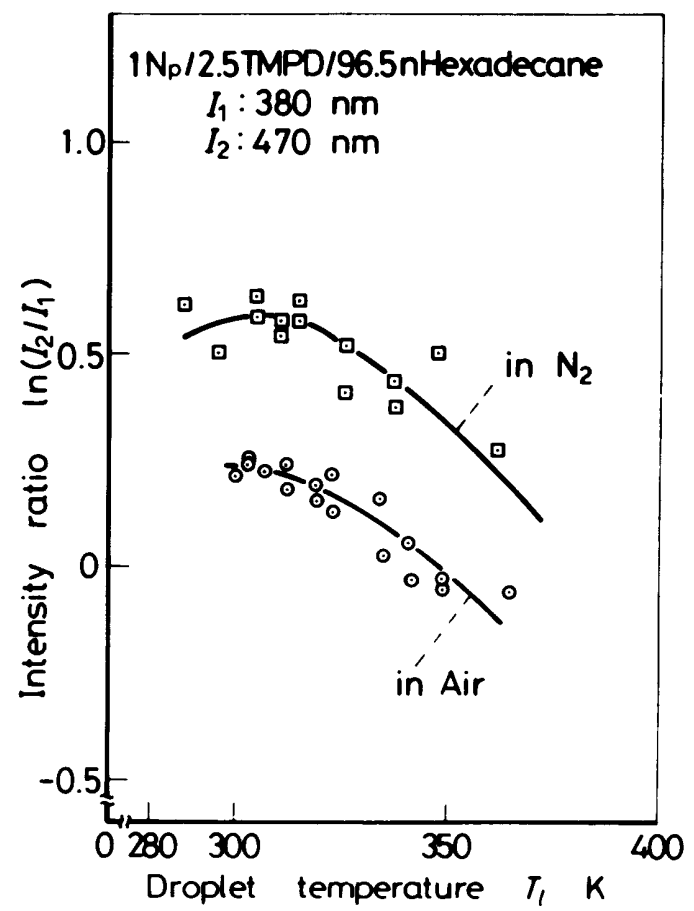

Fig. 7 Ratio of fluorescence emission intensities for $n$ Hexadecane droplet 
appropriate wavelengths at which the fluorescence emission intensities are monitored. Care should be directed to the fact that the relationship between droplet temperature and intensity ratio are affected by the kind of ambient gas.

Figure 8 shows the intensity ratio of fluorescence emission plotted as a function of $n$-Decane droplet temperature. The intensity ratio peaks at a droplet temperature of $320 \mathrm{~K}$ in Nitrogen and at $340 \mathrm{~K}$ in air. Figure 9 shows the results for $n$-Heptane droplets. The intensity ratio peaks at around $300 \mathrm{~K}$. The comparison of the results shown in Figs. 7, 8, and 9 indicates that the peak of the intensity ratio is topped by the $n$-Heptane droplet followed by $n$-Decane and then $n$-Hexadecane droplets in the Nitrogen stream.

Figure 10 shows the intensity ratio for gasoline droplets with no dopant evaporating in the air stream. As anticipated, gasoline droplet thermometry was possible even with no dopant although the sensitivity was rather low. The sensitivity in the thermometry might be improved by selecting two different appropriate wavelengths at which fluorescence emission intensities are monitored. The fact that no dopant is needed in gasoline droplet thermometry is of significance from the practical point of view.

Further studies planned are aimed at improving the sensitivity and accuracy of thermometry by selecting an appropriate concentration of Naphthalene or TMPD doped in the liquid fuel and two different wavelengths at which fluorescence emission intensities

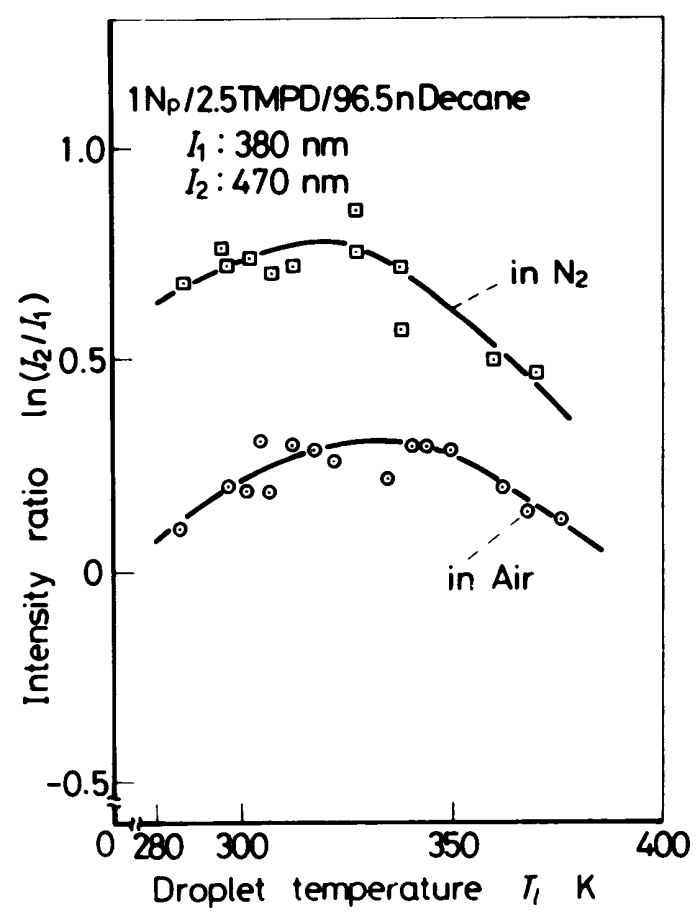

Fig. 8 Ratio of fluorescence emission intensities for $n^{-}$ Decane droplet are monitored for various combinations of fuels and ambient gases.

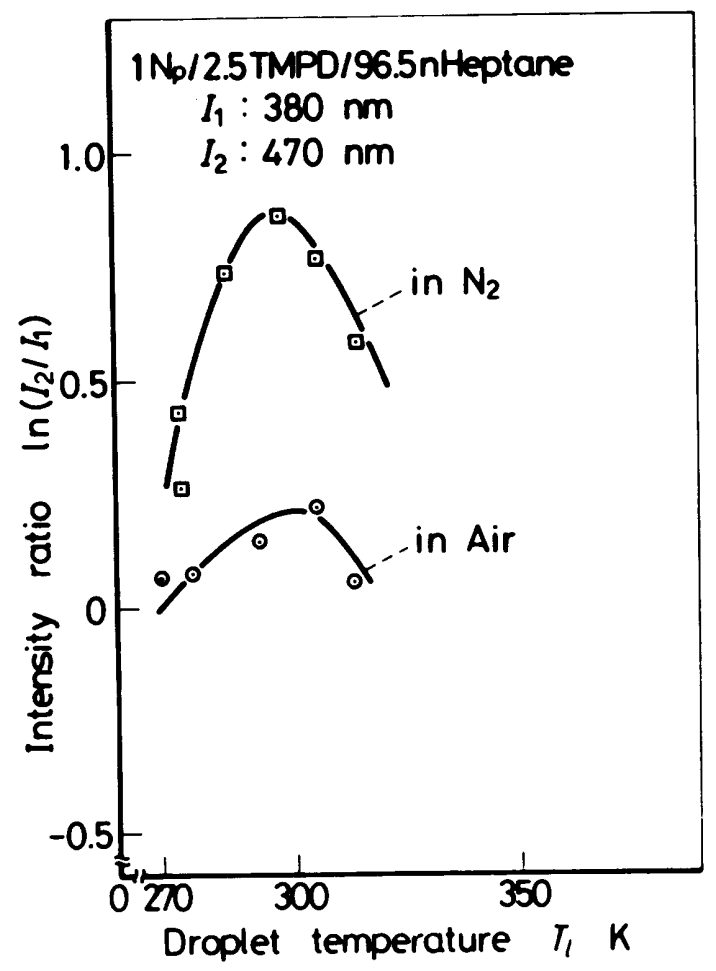

Fig. 9 Ratio of fluorescence emission intensities for $n^{-}$ Heptane droplet

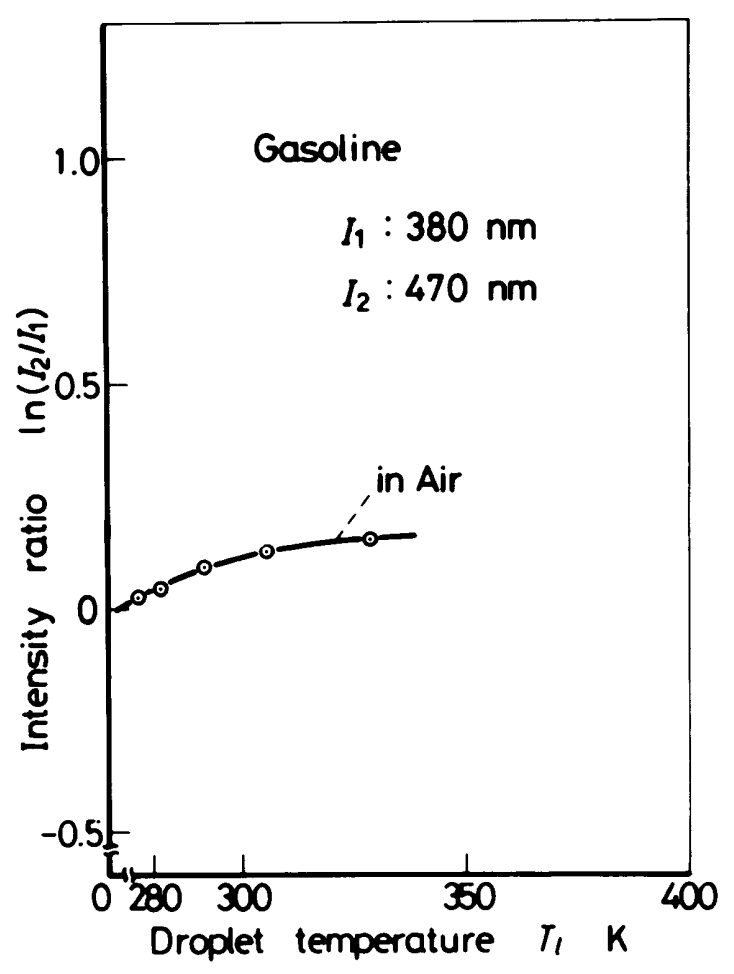

Fig. 10 Ratio of fluorescence emission intensities for gasoline droplet 


\section{Conclusions}

An experimental study was made to develop a diagnostic technique which is available for remote, nonintrusive, instantaneous and point thermometry of liquid fuel droplet by applying the exciplex-based fluorescence method. A suspended droplet doped with $1 \%$ of Naphthalene and $2.5 \%$ of TMPD was allowed to evaporate in a free stream of hot air or Nitrogen, and the fluorescence emission spectra from a droplet subjected to Nitrogen laser excitation were measured.

The primary conclusions reached in the present study are as follows.

(1) The determination of droplet temperature is possible from the measured results of the ratio of fluorescence emission intensities at two different wavelengths, $380 \mathrm{~nm}$ and $470 \mathrm{~nm}$.

(2) The temperature dependence of the intensity ratio is affected by the kind of fuel and ambient gas.

(3) No dopant is needed in gasoline droplet thermometry.

\section{Acknowledgements}

The authors wish to express their gratitude for the financial support of the Tanikawa Foundation.

\section{References}

(1) Williams, A., Combustion of Droplets of Liquid Fuels : A Review, Combust. Flame, Vol. 21, No. 1 (1973), p. 1.

(2) Faeth, G. M., Current Status of Droplet and Liquid Combustion, Prog. Energy Combust. Sci., Vol. 3 (1977), p. 191.

(3) Law, C. K., Recent Advances in Droplet Vaporization and Combustion, Prog. Energy Combust. Sci., Vol. 8(1982), p. 171.

(4) Sirignano, W. A., Fuel Droplet Vaporization and Spray Combustion Theory, Prog. Energy Combust. Sci., Vol. 9(1983),p. 291.

(5) Kadota, T., Ohta, Y. and Sumida, O., Evaporation of the Array of Hydrocarbon Droplets in an Air Stream, Trans. Jpn. Soc. Mech. Eng., (in Japanese), Vol. 54, No. 503, B(1988), p. 1843.

(6) Murray, A. M. and Melton, L. A., Fluorescence Methods for Determination of Temperature in Fuel Sprays, Appl. Opt., Vol. 24, No. 17(1985), p. 2783.

( 7 ) Gossage, H. E. and Melton, L. A., Fluorescence Thermometers Using Intramolecular Exciplexes, Appl. Opt., Vol. 26, No. 11 (1987), p. 2256. 